\title{
Indigenous Knowledge of Herbal Medicines among Adolescents in Amassoma, Bayelsa State, Nigeria
}

\author{
Gideon O. Alade ${ }^{1}$, Ese Okpako ${ }^{1}$, Kola' K. Ajibesin ${ }^{1} \&$ Olanrewaju R. Omobuwajo ${ }^{1}$ \\ ${ }^{1}$ Department of Pharmacognosy \& Herbal Medicine, Faculty of Pharmacy, Niger Delta University, Wilberforce \\ Island, Bayelsa State, Nigeria \\ Correspondence: Gideon O. Alade, Department of Pharmacognosy \& Herbal Medicine, Faculty of Pharmacy, \\ Niger Delta University, Wilberforce Island, Bayelsa State, Nigeria. Tel: 234-806-736-8038. E-mail: \\ aladegideon@yahoo.com, gideon.alade@ndu.edu.ng
}

Received: September 22, 2014 Accepted: October 24, 2014 Online Published: May 20, 2015

doi:10.5539/gjhs.v8n1p237

URL: http://dx.doi.org/10.5539/gjhs.v8n1p237

\begin{abstract}
Background: The use of herbal medicines in Nigeria is on the increase. Documented Population based data on the use of herbal medicinal products and indigenous knowledge among the younger generations are lacking in Nigeria and Africa at large.

Aim: The aim of this study is to investigate the extent of use and general knowledge of herbal medicines among adolescents in the Niger Delta Region of Nigeria.

Methods: The study covered a total of Two hundred and twenty-eight adolescents randomly selected in Senior Secondary Schools (SSS 1-3) in Amassoma using a semi structured questionnaire/Interview and informal conversation on the respondents.

Findings: Nearly all (97\%) the respondents have had contact with herbs. Less than $1 \%$ had contact with herbs through formal education (teachers/literatures). Stimulation of interest was majorly through parents (53\%). Grandparents were the highest (46\%) of custodian of indigenous knowledge. Parents were the next (39.7\%). Only $39 \%$ of the respondents would prefer the use of herbal medicine to modern medicine. Fever was the main ailment mentioned followed by eye ailment and stomach ache. Vernonia amygdalina was the main plant for the treatment of fever.

Conclusion: The study revealed that parents are the major custodians of knowledge being transferred to the younger generation and little or none is learnt from Schools. There is therefore the need to include the study of herbal medicines in School's curricula especially at SS 2 and SS 3 since they are matured enough to appreciate the importance of Herbal medicine so as to prepare them for the promotion of herbal medicine in future and to preserve our indigenous knowledge.
\end{abstract}

Keywords: herbal medicines, indigenous knowledge, secondary schools, Niger Delta

\section{Introduction}

Herbal medicines are drugs made from herbs or plants. They are also commonly referred to as phytomedicines, plant medicines, green medicines, traditional medicine potions, traditional remedies, plant drugs and forest health products among others (Osemene, 2011, Elujoba, 1998). They are also referred to as finished labelled medicinal products that contain as active ingredients aerial or underground parts of plants or other plant materials or combinations thereof whether in the crude state or as plant preparations (WHO, 1996). Plant products include juices, gums, fatty oils and other secondary metabolites such as alkaloids, flavonoids, anthraquinones, saponins among others. They may also contain standard excipients in addition to the active ingredients. Exceptionally, in some countries herbal medicine may also contain by tradition, natural organic or inorganic active ingredients which are not of plant origin (WHO, 1996). Over $80 \%$ of people living in developing countries depend on herbal medicines as their immediate choice in the treatment of diseases showing its relevance and importance in Primary Health Care (Moody, 2007). In 1976, about a quarter of the prescription drugs dispensed by community pharmacy in the United States contained at least one active ingredient derived from plants (Farnsworth \& Morries, 1976). Currently, dispensing herbs/active ingredients is on the increase as herbal medicine is becoming more popular (Ekor, 2013). The WHO estimate of population that has used some 
form of alternative or complementary medicine including Ayurvedic, homeopathic, naturopathic, traditional oriental and Native American Indian medicine in developing countries is between 70 and $80 \%$ (Oreagba \& Oshikoya, 2011).

Herbal medicines have been recognised by the WHO as the most popular form of traditional medicine, and thus, highly lucrative in the international medicine market. Annual revenues in Western Europe were estimated at US\$ 5 billion in 2003-2004, in China the revenue was estimated at US\$ 14 billion in 2005, and in Brazil it was US\$ 160 million in 2007 (Oreagba \& Oshikoya, 2011). The increasing widespread use of Traditional medicine has prompted the WHO to promote its integration into the national health care systems of some countries and to encourage the development of national policy and regulations as essential indicators of the level of integration of such medicine into a national health care system (Oreagba \& Oshikoya, 2011). Also in Nigeria, approximately 205 medicinal plant species are prevalent in nature (FEPA, 1992). Traditional medicine in Nigeria is as old as the people and it is growing in importance and this has made the Federal Government of Nigeria to formulate a traditional medicine policy and to establish a Traditional Medicine Council to regulate practice and encourage research in five core areas (herbal medicine, bone setting, mental health, traditional birth attendance and sale of traditional medicine ingredients (Chesa, 2006). Herbal medicines are by far less concentrated, less toxic and are used in much lower doses than orthodox medicine which in its concentrated drug formulations are designed to target and reverse specific pathologies in the minimum of time (Osemene, 2011; Ohuabunwa, 1998; Moody, 2007). The plants used in herbal medicine have been found to carry their own in-built safety mechanisms. Furthermore, they are ideal tools to restore damaged physiological processes since they consist of a multiplicity of chemical components which act synergistically to make active constituents bio available or to buffer the otherwise potentially powerful active principles thus preventing harmful side effects (Osemene, 2011, Moody, 2007). Herbal medicine has its root in prehistory making every bit as ancient tradition as farming or cooking. In the Graeco-Roman era, Hippocrates (father of medicine), Theophrastus (father of Botany), Galen (originator of pharmaceutical galenicals) and Dioscoroides were all herbalists (Osemene, 2011; Moody, 2007). Globally, people developed unique indigenous healing traditions adapted and defined by their culture, beliefs and environment, which satisfied the health needs of their communities over centuries (Oreagba \& Oshikoya, 2011). Previous studies of herbal medicine use in Nigeria were focused on adults with various forms of chronic illnesses (Danesi \& Adetunji, 1994; Amira \& Okubadejo, 2007; Ogbera et al., 2010), pregnant women (Fakeye et al., 2009) and children with chronic illnesses (Oshikoya et al., 2008) and among a general population without chronic health conditions. No study has ever been evaluated in Nigeria or other African countries on younger population to know if there is transfer of knowledge of herbal medicine from the older population to the younger ones of age range between 14 and 18 years. This study was therefore aimed to assess the extent of use and the general knowledge, benefits and safety of herbal medicines among Senior Secondary School Students resident in Amassoma in the Niger Delta region of Nigeria.

\section{Methodology}

\section{Description of Study Area}

Amassoma is the head quarter of Ogboin clan as well as Ogboin-North Rural Development Authority in the Southern Ijaw Local Government Area of Bayelsa State (Figure 1). It is the host community to the Niger Delta University, Wilberforce Island Bayelsa. It is located about $40 \mathrm{~km}$ to the South of Yenagoa; the State capital. It is on an altitude of 512 above sea level, bounded in the North by River Nun, West by Otuan, East by Toru Ebeni and the South by Ogobiri. It is the biggest town in Southern Ijaw Local Government Area. The area has a coastline of approximately $60 \mathrm{~km}$ on the Bight of Bonny. It has an area of 2,682 $\mathrm{km}^{2}$ and a population of 319,413 (Federal Government of Nigeria, 2007) 


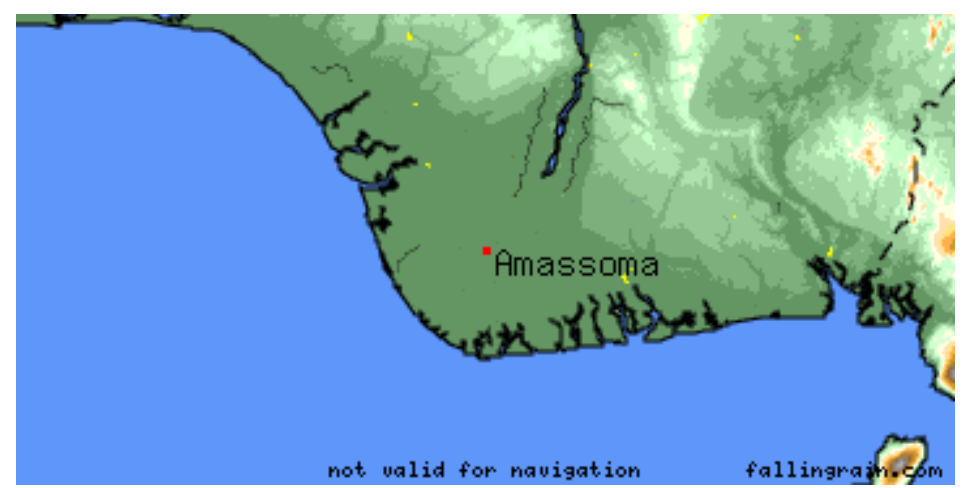

Figure 1. Map of Southern Ijaw showing Amassoma

The study covered the only two Secondary Schools having Senior (SS 1 to SS 3) classes in Amassoma. Students in the Senior Secondary classes were involved. Permission was sought from the School management. A total of Two hundred and twenty-eight (228) students were present in these classes. Only 164 (72\%) students responded and finally selected for the study through a purposive and convenience sampling process. Furthermore, semi structured questionnaires/ interviews amidst informal conversation on the respondents whose opinions were sought on their knowledge of herbal medicine. The questionnaires had two components: Demography and Indigenous knowledge on herbal medicines. The participants were allowed to give the vernacular names of the plants while some were identified at site. The results of the pre-tested questionnaires were used to make necessary modifications and corrections on the questionnaires and interview guides. Data were analysed using descriptive statistics such as frequency and percentages.

\section{Results and Discussion}

\section{Demography}

Males were more in all the three arms of the classes with $54 \%$ except in S. S. S 2 with a higher number of females (54\%). It has been reported that Bayelsa state is among the States in Nigeria with low girl child education probably due to under age child bearing and poverty (Punch Newspaper, Oct., 2013). Age range of 14 - 18 years was the highest in terms of the number of respondents ( $86 \%$ of students in this category) and the majority $(82 \%)$ speaks Ijaw language apart from English which is the medium of communication in Nigerian schools (Table 1).

Table 1. Demographical Data of the study Population

\begin{tabular}{lllll}
\hline & SS1 & SS2 & SS3 & TOTAL \\
\hline GENDER & & & & \\
MALE & 50 & 19 & 20 & $89(54 \%)$ \\
FEMALE & 35 & 21 & 19 & $75(46 \%)$ \\
AGE & & & & \\
9 TO 13 & 7 & 1 & 1 & $9(6 \%)$ \\
14 TO 18 & 75 & 37 & 30 & $142(86 \%)$ \\
$>19$ & 3 & 2 & 8 & $13(8 \%)$ \\
LANGUAGE & & & & \\
IJAW & 69 & 36 & 30 & $135(82.2 \%)$ \\
YORUBA & 0 & 0 & 2 & $2(1.2 \%)$ \\
IBO & 5 & 0 & 0 & $5(3.1 \%)$ \\
HAUSA & 0 & 0 & 0 & $0(0 \%)$ \\
OTHERS & 2 & 1 & 2 & $5(3.1 \%)$ \\
COMBINATION & 9 & 3 & 5 & $17(10.4 \%)$ \\
\hline
\end{tabular}




\subsection{Contact With Herbs}

Nearly all the respondents $(97 \%)$ have had contact with herbs. The highest medium of contact was through usage (approximately 40\%), 25\% acquired knowledge on herbs through their parents. This is different from a report in which $80 \%$ acquired their knowledge through parents among Secondary and Grammar School students in Slovenia (Strgar et al., 2013). While $37 \%$ had contact through a combination of usage, parents, Teachers and media, only less than $1 \%$ had it through their teachers/literature. This agrees with the case in Slovenia in which it was found that little or nothing on the subject was learnt in school (Strgar et al., 2013). Their stimulation of interest was majorly from their parents $(53 \%)$, followed by their Grand parents $(22 \%)$. Others were through combinations of parents, grandparents, herbalists and other relations. This showed that parents are still eager to transfer their indigenous knowledge to their children. Twenty percent of respondents believed that their interest in herbal medicine is high, while majority (52\%) showed a middle rating in their interest and $27 \%$ showed low interest. Approximately $90 \%$ has used herbal medicines at one time or the other whether once, a few times, frequently or occasionally, while only $10 \%$ have never taken it before. Out of the $90 \%$, about $26 \%$ takes it frequently. Out of these $90 \%$ users, approximately $70 \%$ are highly satisfied with the effectiveness of herbal medicine.The remaining had a low satisfaction (31\%) (Table 2).

Table 2. Contact of Respondents to herbal medicines

\begin{tabular}{|c|c|c|c|c|}
\hline & SS 1 & SS 2 & SS 3 & TOTAL \\
\hline \multicolumn{5}{|l|}{ CONTACT WITH HERBS } \\
\hline YES & 80 & 40 & 39 & $159(97 \%)$ \\
\hline NO & 5 & 0 & 0 & $5(3.1 \%)$ \\
\hline \multicolumn{5}{|l|}{ MEDIUM OF CONTACT } \\
\hline USAGE & 23 & 13 & 27 & $63(39.6 \%)$ \\
\hline PARENTS & 10 & 5 & 25 & $40(25.2 \%)$ \\
\hline TEACHERS/LITERATURE & 1 & 0 & 0 & $1(0.6 \%)$ \\
\hline MEDIA & 11 & 3 & 0 & $14(8.8 \%)$ \\
\hline OTHERS & 3 & 1 & 0 & $4(2.5 \%)$ \\
\hline COMBINATION & 27 & 10 & 0 & $37(23.3 \%)$ \\
\hline \multicolumn{5}{|l|}{ STIMULATION OF INTEREST } \\
\hline PARENTS & 48 & 19 & 20 & $87(53 \%)$ \\
\hline GRAND PARENTS & 17 & 9 & 10 & $36(22 \%)$ \\
\hline RELATIONS & 4 & 3 & 2 & $9(5.5 \%)$ \\
\hline HERBALIST & 0 & 0 & 1 & $1(0.6 \%)$ \\
\hline OTHERS & 6 & 6 & 6 & $18(11 \%)$ \\
\hline COMBINATION & 10 & 3 & 0 & $13(7.9 \%)$ \\
\hline \multicolumn{5}{|l|}{ NONE } \\
\hline \multicolumn{5}{|l|}{ LEVEL OF INTEREST } \\
\hline LOW & 24 & 11 & 10 & $45(27.4 \%)$ \\
\hline MIDDLE & 41 & 20 & 25 & $86(52.4 \%)$ \\
\hline HIGH & 20 & 9 & 4 & $33(20.1 \%)$ \\
\hline NONE & 0 & 0 & 0 & $0(0 \%)$ \\
\hline \multicolumn{5}{|c|}{ NO OF TIMES HERBS HAVE BEEN TAKEN } \\
\hline ONCE & 22 & 2 & 7 & $31(19.5 \%)$ \\
\hline FEW & 16 & 13 & 22 & $51(32.1 \%)$ \\
\hline FREQUENT & 18 & 18 & 6 & $42(26.4 \%)$ \\
\hline
\end{tabular}




\begin{tabular}{lllll} 
OCCASSIONAL & 19 & 2 & 0 & $21(13.2 \%)$ \\
NONE & 8 & 2 & 4 & $14(8.8 \%)$ \\
LEVEL OF SATISFACTION & & & & \\
LOW & 23 & 8 & 14 & $45(31 \%)$ \\
HIGH & 50 & 28 & 22 & $100(69 \%)$ \\
NONE & 0 & 0 & 0 & $0(0 \%)$ \\
\hline
\end{tabular}

\subsection{Knowledge of Medicinal Plants}

Grandparents were the highest (46\%) custodian of knowledge on medicinal plants in their family followed by their parents with about $40 \%$. Out of the latter, mothers had more knowledge on medicinal plants than fathers with approximately $60 \%$. Women have been recognized as users and custodians of plant biodiversity. In countries like Bolivia, Colombia, Peru, Viet Nam, Indonesia and India, they are responsible for the selection, improvement and storage of seeds and management. Women from sub-Saharan Africa grow over 120 different plant varieties in small areas alongside cash crops (Deda \& Rubian, 2004). In a study in Sierra Leone, women could name 31 uses of trees on fallow land and in the forest, while men named eight different uses (Aguilar, 2004). Women have a unique relationship with biodiversity across the globe; they predominate as wild plant gatherers, home gardeners, plant domesticators, herbalists and seed custodians.

Majority (55\%) would prefer modern medicine to herbal medicines $(39 \%)$, while $6 \%$ would prefer to opt for both since from their perception, they work synergistically. Out of the $39 \%$ that preferred herbal medicine, $61 \%$ of them would prefer herbal medicine for its effectiveness, affordability, accessibility and safety. Only $43 \%$ would like to attend a course on herbal medicine if there was an opportunity as against about $94 \%$ in the case of Slovenia probably because they think herbal medicine is enmeshed in esoterism or does not yet enjoy official recognition by Government. Thirty-one percent (31\%) gave a positive response that they would like to practise herbal medicine. A previous report has shown that the younger generation does not seem to have much trust in the traditional medicine system which may be attributed to increasing use of allelopathic medicines which are readily available and considered potent. Up to $85 \%$ of respondents had knowledge of medicinal plants and about $95 \%$ of these medicinal plants mentioned grow or are cultivated around the home. Approximately $90 \%$ could identify the common plants growing around them, $83.5 \%$ of them can prepare them for use and up to $90 \%$ of these has at one time or the other been involved in self-treatment or prescription (Table 3).

Table 3. Knowledge of herbal medicine by the Respondents

\begin{tabular}{lllll}
\hline & SS 1 & SS 2 & SS 3 & TOTAL \\
\hline BEST KNOWLEDGE OF MEDICINAL PLANTS IN THE FAMILY & & & & \\
MOTHER & 17 & 10 & 11 & $38(24 \%)$ \\
FATHER & 11 & 4 & 10 & $25(15.7 \%)$ \\
GRAND PARENTS & 45 & 16 & 12 & $73(46 \%)$ \\
OTHERS & 8 & 6 & 3 & $17(10.7 \%)$ \\
COMBINATION & 1 & 3 & 0 & $4(2.5 \%)$ \\
NONE & 0 & 0 & 3 & $3(1.9 \%)$ \\
PREFERENCE OF MEDICINE & & & & \\
HERBAL MEDICINE & 35 & 16 & 13 & $64(39 \%)$ \\
MODERN MEDICINE & 46 & 21 & 23 & $90(55 \%)$ \\
EQUAL & 4 & 3 & 2 & $9(5.5 \%)$ \\
NONE & 0 & 0 & 1 & $1(0.6 \%)$ \\
REASON & & & & \\
HERBAL MEDICINE & & & & \\
EFFECTIVE & 12 & 8 & 7 & $27(42.2 \%)$
\end{tabular}




\begin{tabular}{|c|c|c|c|c|}
\hline AFFORDABLE & 2 & 1 & 0 & $3(4.7 \%)$ \\
\hline ACCESSIBLE & 4 & 1 & 1 & $6(9.4 \%)$ \\
\hline NATURAL AND SAFE & 5 & 2 & 0 & $7(4.3 \%)$ \\
\hline COMBINATION & 12 & 4 & 5 & $21(32.8 \%)$ \\
\hline \multicolumn{5}{|l|}{ MODERN } \\
\hline EFFECTIVE & 27 & 11 & 10 & $48(53.3 \%)$ \\
\hline ACCESSIBLE & 4 & 2 & 1 & $7(7.8 \%)$ \\
\hline SUBJECTED TO SCIENTIFIC RESEARCH & 5 & 2 & 5 & $12(13.3 \%)$ \\
\hline PRESENTABLE & 3 & 2 & 1 & $6(6.7 \%)$ \\
\hline CIVILIZATION & 1 & 0 & 0 & $1(1.1 \%)$ \\
\hline SAFE & 0 & 1 & 1 & $2(2.1 \%)$ \\
\hline COMBINATION & 6 & 3 & 5 & $14(15.6 \%)$ \\
\hline \multicolumn{5}{|l|}{ EQUAL } \\
\hline BOTH CAN WORK TOGETHER & 4 & 3 & 2 & 9 \\
\hline \multicolumn{5}{|l|}{ NONE } \\
\hline RELIGIOUS BELIEF & 0 & 0 & 1 & 1 \\
\hline \multicolumn{5}{|l|}{ COURSE ON HERBAL MEDICINE } \\
\hline YES & 35 & 20 & 15 & $70(42.7 \%)$ \\
\hline NO & 50 & 20 & 24 & $94(57.3 \%)$ \\
\hline \multicolumn{5}{|l|}{ PRACTICE OF TRADITIONAL MEDICINE } \\
\hline YES & 25 & 15 & 10 & $50(30.5 \%)$ \\
\hline NO & 60 & 25 & 29 & $114(69.5 \%)$ \\
\hline \multicolumn{5}{|l|}{ KNOWLEDGE OF MEDICINAL PLANTS } \\
\hline YES & 71 & 35 & 33 & $139(84.8 \%)$ \\
\hline NO & 14 & 6 & 5 & $25(15.2 \%)$ \\
\hline \multicolumn{5}{|l|}{ ARE THEY CULTIVATED AROUND THE HOME } \\
\hline YES & 63 & 35 & 34 & $132(95 \%)$ \\
\hline NO & 6 & 0 & 1 & $7(5 \%)$ \\
\hline \multicolumn{5}{|l|}{ PLANT IDENTIFICATION } \\
\hline YES & 55 & 35 & 34 & $124(89.2 \%)$ \\
\hline NO & 12 & 0 & 3 & $15(10.8 \%)$ \\
\hline \multicolumn{5}{|l|}{ PREPARATION OF HERBAL MEDICINE } \\
\hline YES & 53 & 31 & 32 & $116(83.5 \%)$ \\
\hline NO & 14 & 4 & 5 & $23(16.5 \%)$ \\
\hline \multicolumn{5}{|l|}{ SELF TREATMENT/PRESCRIPTION } \\
\hline YES & 48 & 30 & 26 & $104(90 \%)$ \\
\hline NO & 2 & 5 & 5 & $12(10 \%)$ \\
\hline
\end{tabular}

\subsection{Plant Species}

Fever was the main ailment treated with Vernonia amygdalina, Carica papaya and Citrus $x$ aurantifolia while Eye ailment was for Ocimum gratissimum and Telfairia occidentalis is for boosting blood (Table 4). When the plant species were categorized into 29 families, it was observed that the most cited one was Asteraceae (10.26\%), followed by the family Euphorbiaceae (7.69\%) (Table 5). These two families have also been reported to be 
predominant in an ethnobotanical inventories carried out in some Southern parts of Nigeria (Uzodimma, 2013, Obata \& Aigbokhan, 2012).

Table 4. Medicinal plants mentioned by the students and their uses

\begin{tabular}{|c|c|c|c|c|}
\hline & Plant & Family & Common names & Main disease \\
\hline 1 & Acalypha wikesiana Muell Arg & Euphorbiaceae & Acalypha & Skin infection \\
\hline 2 & Aframomum melegueta K. Schum & Zingiberaceae & Alligator pepper & Wound \\
\hline 3 & Ageratum conyzoides L. & Asteraceae & Goat weed & Eye \\
\hline 4 & Allium cepa $\mathrm{L}$. & Alliaceae & Onion & Fainting \\
\hline 5 & Aloe vera $(\mathrm{L}$.$) Burm.f$ & Aloaceae & Aloe vera & Eye \\
\hline 6 & Ananas comosus Merr. & Bromeliaceae & Pineapple & Measles \\
\hline 7 & Azdirachta indica A. Juss & Meliaceae & Neem & Fever \\
\hline 8 & Bryophyllum pinnatum (Lam) Oken & Crassulaceae & Never die & Cough \\
\hline 9 & Capsicum frutescens $\mathrm{L}$. & Solanaceae & Pepper & Wound \\
\hline 10 & Carica papaya $\mathrm{L}$. & Caricaceae & Pawpaw & Fever \\
\hline 11 & $\begin{array}{l}\text { Chromolaena odorata (L.) King \& H.E } \\
\text { Robins }\end{array}$ & Asteraceae & Christmas bush & Bleeding \\
\hline 12 & Citrus $x$ aurantifolia Burn.f. & Myrtaceae & Lime & Fever \\
\hline 13 & Citrus $x$ sinensis Osbeck & Rutaceae & Orange & Energy/appetite \\
\hline 14 & Cola nitida (Vent.) Schott \&Endl. & Sterculiaceae & Kola nut & Skin infection \\
\hline 15 & Corchorus olitorius L. & Tiliaceae & Jute leaf & Skin beauty \\
\hline 16 & Costus afer Ker Gawl & Costaceae & Monkey sugar cane & Chicken pox \\
\hline 17 & Cymbopogon citratus DC Stapf. & Poaceae & Lemon grass & Fever \\
\hline 18 & Elaeis guineensis Jacq. & Arecaceae & Palm kernel & Fever \\
\hline 19 & Ficus exasperata $\mathrm{L}$ & Moraceae & Fig tree & Blood \\
\hline 20 & Garcinia kola Heckel & Clusiaceae & Bitter cola & Cough \\
\hline 21 & Helianthus anuus $\mathrm{L}$ & Asteraceae & sun flower & Bleeding \\
\hline 22 & Hibiscus esculentus (L.) Moench & Malvaceae & Okro & Bite \\
\hline 23 & Ipomea batatas $\mathrm{L}$. & Convovulaceae & Potato & Pile \\
\hline 24 & Jatropha tanjorensis Ellis \&Saroja & Euphorbiaceae & Hospital too far & Blood \\
\hline 25 & Solanum lycopersicum $\mathrm{L}$ & Solanaceae & Tomato & Blood \\
\hline 26 & Mangifera indica $\mathrm{L}$. & Anacardiaceae & Mango & Fever \\
\hline 27 & Manihot esculenta Crantz & Euphorbiaceae & Cassava & Bite/inflammation \\
\hline 28 & Moringa oleifera $\mathrm{L}$. & Moringaceae & Moringa & Eye \\
\hline 29 & Musa paradisiaca $\mathrm{L}$. & Musaceae & Plantain & Chicken pox \\
\hline 30 & Ocimum gratissimum $\mathrm{L}$. & Lamiaceae & Scent leaf & Eye \\
\hline 31 & Pennisetum purpureum $\mathrm{L}$ & Poaceae & Elephant grass & Fever \\
\hline 32 & Persea americana Mill & Lauraceae & Avocadro pear & Arthritis \\
\hline 33 & Phyllanthus amarus Schum. \& Thonn. & Phyllathaceae & Phyllanthus & Labour induction \\
\hline 34 & Psidium guajava $\mathrm{L}$. & Rutaceae & Guava & Stomach ache \\
\hline 35 & Talinium trangulare (Jacq.) Willd. & Portulacaceae & Water leaf & Pain \\
\hline 36 & Telfairia occidentalis Hook. F & Cucurbitaceae & Fluted pumpkin & Blood \\
\hline 37 & Tetrapleura tetraptera Taub. & Leguminosae-Mimosaceae & Tetrapleura & Ulcer \\
\hline
\end{tabular}




\begin{tabular}{|c|c|c|c|c|}
\hline 38 & Uvaria chamae P. Beauv. & Annonaceae & Bush pepper & Skin infection \\
\hline 39 & Vernonia amygdalina Delile & Asteraceae & Bitter leaf & Fever \\
\hline & OTHERS & & & \\
\hline 40 & & & Cray fish & Blood \\
\hline 41 & & & Fresh egg & Blood \\
\hline 42 & & & Honey & Arthritis \\
\hline 43 & & & Male lizard & Cough \\
\hline 44 & & & Snake & Skin rashes \\
\hline
\end{tabular}

Table 5. Medicinal Plant families mentioned by the students

\begin{tabular}{|c|c|c|}
\hline Families & Occurrence & $\%$ Occurrence \\
\hline Aliaceae & 1 & 2.56 \\
\hline Aloaceae & 1 & 2.56 \\
\hline Anarcadaceae & 1 & 2.56 \\
\hline Annonaceae & 1 & 2.56 \\
\hline Arecaceae & 1 & 2.56 \\
\hline Asteraceae & 4 & 10.26 \\
\hline Bromeliaceae & 1 & 2.56 \\
\hline Caricaceae & 1 & 2.56 \\
\hline Convovulaceae & 1 & 2.56 \\
\hline Costaceae & 1 & 2.56 \\
\hline Crassulaceae & 1 & 2.56 \\
\hline Cucurbitaceae & 1 & 2.56 \\
\hline Euphorbiaceae & 3 & 7.69 \\
\hline Gutiferae & 1 & 2.56 \\
\hline Lamiaceae & 1 & 2.56 \\
\hline Lauraceae & 1 & 2.56 \\
\hline Leguminosae-Mimosaceae & 1 & 2.56 \\
\hline Malvaceae & 1 & 2.56 \\
\hline Meliaceae & 1 & 2.56 \\
\hline Moraceae & 1 & 2.56 \\
\hline Moringaceae & 1 & 2.56 \\
\hline Musaceae & 1 & 2.56 \\
\hline Myrtaceae & 1 & 2.56 \\
\hline Poaceae & 2 & 5.13 \\
\hline Portulaccaceae & 1 & 2.56 \\
\hline Rutaceae & 2 & 5.13 \\
\hline Solanaceae & 1 & 2.56 \\
\hline Sterculiaceae & 1 & 2.56 \\
\hline Tiliaceae & 1 & 2.56 \\
\hline \multirow[t]{2}{*}{ Zingiberaceae } & 1 & 2.56 \\
\hline & 39 & \\
\hline
\end{tabular}




\subsection{Disease Category}

Fever was the most frequently mentioned singular disease with $43 \%$ citation having Vernonia amygdalina as the most cited plant species for it (37\%). This agrees with the findings from Portharcourt metropolis in the Niger Delta Region, in which most of the plants cited were used to treat malaria fever, underlying the importance of this disease in the region (Nwazuoma \& Dappa, 2013). Malaria has been reported as the world's most important parasitic disease. Nearly half of her population is exposed to malaria. An estimate of 3.3 billion people was at risk in 2011 with Sub-saharan Africa having the highest risk (Kasali et al., 2014; Ibrahim et al., 2012; Adebayo \& Kretti, 2011). It is endemic in Nigeria, accounting for a quarter of all cases in the Sub-saharan Africa (Omosun et al., 2013), with about $97 \%$ of the population at risk (FMOH, 2009). It also accounted for nearly 110 million clinically diagnosed cases of fever yearly and an estimate of annual infant and children under five mortality of about $25 \%$ and $30 \%$ respectively $(\mathrm{FMOH}, 2009)$. It is also responsible for an estimated maternal mortality of $11 \%$. Apart from the direct health impact, the annual social and economic burden in form of treatment cost, prevention cost and loss of man hours, is estimated to be about 132 billion naira (Kunle et al., 2013). The Bayelsa State Ministry of Health has also identified malaria as the lead cause of 210 deaths recorded in the State in 2011. Out of the 210 cases of deaths that occurred in about 35 different diseases under the public health sector surveillance, malaria was said to have led the pack with 102 deaths in 2011 (Daily Trust, Feb. 28, 2012). Niger Delta Region experiences the highest amounts of rain fall in Nigeria receiving over $4000 \mathrm{~mm}$ (157.5 in) annually thus the terrain is characterized by marshy areas which encourage the breeding of mosquitoes. Next to fever in rank was eye ailment (9.1\%), the most cited plant species for it was Ocimum gratissimum (10.8\%). These were followed by Blood diseases (6.6\%), Stomach ache (7\%), Bleeding (4\%) with the most cited plant species as Telfairi aoccidentalis (8.7\%), Citrus aurantifolia (3.5\%) and Vernonia amygdalina (6.9\%) respectively (Table 6, Appendices 1a, b, and c). When the diseases were categorized, fever ranked highest among the categories of diseases cited by the respondents $(45 \%), 13.5 \%$ of plant species were mentioned for this category. Next to it was haematological conditions which had 13\% citation and $13.5 \%$ plant species mentioned for it. Others were gastrointestinal disorders (10.6\%), ophthalmology (9.4\%) and musculoskeletal ailments $(6.4 \%)$ with $15 \%, 6.8 \%$ and $13.5 \%$ number of species of plants cited respectively (Table 7).

Table 6. Category of ailments and most cited medicinal plants for treatment

\begin{tabular}{|c|c|c|c|c|c|c|}
\hline $\mathrm{s} / \mathrm{n}$ & Disease & Citation & $\begin{array}{l}\% \text { citation } \\
\text { of disease }\end{array}$ & most cited plant (s) & $\begin{array}{l}\text { citation of } \\
\text { plant for } \\
\text { Disease }\end{array}$ & $\begin{array}{l}\% \text { citation of } \\
\text { plant for } \\
\text { Disease }\end{array}$ \\
\hline 1 & Acne & 2 & 0.4 & Citrus aurantifolia & 2 & 0.9 \\
\hline \multirow[t]{2}{*}{2} & Apetite & 2 & 0.4 & Citrus sinensis & 1 & 0.4 \\
\hline & & & & Capsicum frutescens & 1 & 0.4 \\
\hline \multirow[t]{2}{*}{3} & Arthritis & 3 & 0.6 & Ocimum gratissimum & 1 & 0.4 \\
\hline & & & & Persea americana & 1 & 0.4 \\
\hline 4 & Asthma & 1 & 0.2 & Allium cepa & 1 & 0.4 \\
\hline 5 & Bleeding & 19 & 4 & Vernonia amygdalina & 16 & 6.9 \\
\hline 6 & Blood & 31 & 6.6 & Telfairia occidentalis & 20 & 8.7 \\
\hline 7 & Body tonic & 2 & 0.4 & Ocimum gratissimum & 1 & 0.4 \\
\hline 8 & Catarrh & 6 & 1.3 & Ocimum gratissimum & 3 & 1.3 \\
\hline \multirow[t]{3}{*}{9} & Chicken pox & 3 & 0.6 & Musa paradisiaca & 1 & 0.4 \\
\hline & & & & Azadirachta indica & 1 & 0.4 \\
\hline & & & & Costus afer & 1 & 0.4 \\
\hline 10 & Conjunctivitis & 1 & 0.2 & Musa paradisiaca & 1 & 0.4 \\
\hline 11 & Cough & 11 & 2.3 & Garcinia kola & 2 & 0.9 \\
\hline 12 & Diabetes & 4 & 0.9 & Vernonia amygdalina & 3 & 1.3 \\
\hline 13 & Diarrhoea & 3 & 0.6 & Vernonia amygdalina & 1 & 0.4 \\
\hline
\end{tabular}




\begin{tabular}{|c|c|c|c|c|c|c|}
\hline & & & & Ocimum gratissimum & 1 & 0.4 \\
\hline \multirow[t]{2}{*}{14} & Dislocation & 1 & 0.2 & Capsicum frutescens & 1 & 0.4 \\
\hline & & & & Aframomum melegueta & 1 & 0.4 \\
\hline 15 & Dry throat & 2 & 0.4 & Capsicum frutescens & 1 & 0.4 \\
\hline 16 & Ear infection & 2 & 0.4 & Bryophyllum pinnatum & 1 & 0.4 \\
\hline 17 & Eczema & 4 & 0.9 & Bryophyllum pinnatum & 4 & 1.7 \\
\hline 18 & Energy & 5 & 1.1 & Telfairia occidentalis & 2 & 0.9 \\
\hline 19 & Eye & 43 & 9.1 & Ocimum gratissimum & 25 & 10.8 \\
\hline 20 & Fainting & 1 & 0.2 & Allium сера & 2 & 0.9 \\
\hline 21 & Headache & 9 & 1.9 & Ocimum gratissimum & 7 & 3 \\
\hline 22 & Hernia & 1 & 0.2 & Tetrapleura tetraptera & 1 & 0.4 \\
\hline 23 & High Blood Pressure & 3 & 0.6 & Moringa oleifera & 2 & 0.9 \\
\hline \multirow[t]{2}{*}{24} & Inflammation & 4 & 0.9 & Ocimum gratissimum & 1 & 0.4 \\
\hline & & & & Garcinia kola & 1 & 0.4 \\
\hline 25 & Labour induction & 1 & 0.2 & Phyllanthus amarus & 2 & 0.9 \\
\hline 26 & Malaria & 202 & 43 & Vernonia amygdalina & 85 & 36.8 \\
\hline 27 & Massaging & 1 & 0.2 & Elaeisis guineensis & 1 & 0.4 \\
\hline 28 & Measles & 6 & 1.3 & Vernonia amygdalina & 3 & 1.3 \\
\hline 29 & Menstrual pain & 1 & 0.2 & Musa paradisiaca & 1 & 0.4 \\
\hline 30 & Navel healing & 1 & 0.2 & Bryophyllum pinnatum & 1 & 0.4 \\
\hline \multirow[t]{2}{*}{31} & Pain & 11 & 2.3 & Citrus aurantifolia & 2 & 0.9 \\
\hline & & & & Moringa oleifera & 2 & 0.9 \\
\hline 32 & Pile & 5 & 1.1 & Ipomea batatas & 4 & 1.7 \\
\hline 33 & Ringworm & 1 & 0.2 & Aloe vera & 1 & 0.4 \\
\hline \multirow[t]{2}{*}{34} & Skin beauty & 2 & 0.4 & Telfairia occidentalis & 1 & 0.4 \\
\hline & & & & Cochorus olitorius & 1 & 0.4 \\
\hline 35 & Skin infection & 11 & 2.3 & Ocimum gratissimum & 5 & 2.2 \\
\hline \multirow[t]{2}{*}{36} & Snake/ scorpion Bite & 4 & 0.9 & Manihot esculenta & 2 & 0.9 \\
\hline & & & & Hibiscus esculentus & 2 & 0.9 \\
\hline 37 & Stomach ache & 33 & 7 & Citrus aurantifolia & 8 & 3.5 \\
\hline 38 & Stomach ulcer & 6 & 1.3 & Tetrapleura tetraptera & 3 & 1.3 \\
\hline 39 & Typhoid & 11 & 2.3 & Carica papaya & 6 & 2.6 \\
\hline \multirow[t]{3}{*}{40} & Weight control & 3 & 0.6 & Ocimum gratissimum & 1 & 0.4 \\
\hline & & & & Cochorus olitoriu & 1 & 0.4 \\
\hline & & & & Citrus aurantifolia & 1 & 0.4 \\
\hline 41 & Worm expeller & 1 & 0.2 & Citrus aurantifolia & 1 & 0.4 \\
\hline 42 & Wound & 6 & 1.3 & Ocimum gratissimum & 4 & 1.7 \\
\hline
\end{tabular}


Table 7. Categories of Prevalent diseases in the study area

\begin{tabular}{|c|c|c|c|c|c|c|}
\hline & Disease category & citation & $\%$ citation & Number of specie & $\%$ & Number of specie \\
\hline 1 & Cardiovascular diseases & 3 & 0.6 & 2 & 1.5 & \\
\hline 2 & Dermatology & 33 & 7 & 20 & 15 & \\
\hline 3 & Fever & 213 & 45.3 & 18 & 13.5 & \\
\hline 4 & Gastrointestinal tract & 50 & 10.6 & 20 & 15 & \\
\hline 5 & Haematology & 61 & 13 & 18 & 13.5 & \\
\hline 6 & Metabolic diseases & 12 & 2.6 & 9 & 6.8 & \\
\hline 7 & Musculoskeletal & 30 & 6.4 & 18 & 13.5 & \\
\hline 8 & Obstetrics and Gynaecology & 3 & 0.6 & 2 & 1.5 & \\
\hline 9 & Ophthalmology & 44 & 9.4 & 9 & 6.8 & \\
\hline 10 & Respiratory /Ear Nose \& throat & 22 & 4.7 & 15 & 11.3 & \\
\hline \multirow[t]{2}{*}{11} & Structural diseases & 2 & 0.4 & 2 & 1.5 & \\
\hline & & 473 & & 133 & & \\
\hline
\end{tabular}

\subsection{Plant's Habits and Plant Parts Used As Medicines}

Approximately $33 \%$ of the herbal medicines mentioned were from shrub, $30 \%$ were obtained from trees, $27.5 \%$ from herbs while $10 \%$ were shared equally between grass and climbers (Tables 8 and Figure 2). Majority (84\%) of the herbal medicines mentioned were obtained from leaf while root produced the least $(0.2 \%)$ (Figure 3$)$. The use of leaves could be justified by the abundance of chemical groups they contain. In fact, leaves are known as the main synthesis site of secondary metabolites in plants and are the most commonly used plant parts by traditional medicine practitioners (Katemo et al., 2012; Lavergne \& Vera, 1998; Idowu et al., 2010; Pousset, 1989; Moswa, 2005). This also constitutes an advantage as harvesting leaves on a sustainable manner ensures continuity of the plant.

Table 8. Plant habits

\begin{tabular}{lll}
\hline Medicinal palnts & Family & Habits \\
\hline Acalypha wikesiana Muell Arg & Euphorbiaceae & Shrub \\
Aframomum melegueta K. Schum & Zingiberaceae & Herb \\
Ageratum conyzoides L. & Asteraceae & Shrub \\
Allium cepa L. & Aliaceae & Herb \\
Aloe vera (L.) Burm.f & Aloaceae & Shrub \\
Ananas comosus Merr. & Bromeliaceae & Herb \\
Azdirachta indica A. Juss & Meliaceae & Shrub \\
Bryophyllum pinnatum (Lam) Oken & Crassulaceae & Herb \\
Capsicum frutescens L. & Solanaceae & Shrub \\
carica papaya L. & Caricaceae & Tree \\
Chromolaena odorata (L.) King \& H.E Robins & Asteraceae & Shrub \\
Citrus aurantifolia Burn.f & Myrtaceae & Tree \\
Citrus sinensis Osbeck & Rutaceae & Tree \\
Cola nitida (Vent.) Schott \&Endl. & Sterculiaceae & Tree \\
Corchorus olitorius L. & Tiliaceae & Herb \\
Costus afer Ker Gawl & Costaceae & Shrub
\end{tabular}


Cymbopogon citratus DC Stapf.

Elaeis guineensis Jacq.

Ficus exasperata $\mathrm{L}$

Garcinia kola Heckel

Hibiscus esculentus (L.) Moench

Ipomea batatas $\mathrm{L}$.

Jatropha tanjorensis Ellis \& Saroja

Solanum lycopersicum L.

Mangifera indica L.

Manihot esculenta Crantz

Moringa oleifera L.

Musa paradisiaca $\mathrm{L}$.

Ocimum gratissimum L.

Pennisetum purpureum L.

Persea americana Mill

Phyllanthus amarus Schum. \& Thonn.

Psidium guajava L.

Talinium trangulare (Jacq.) Willd.

Telfairia occidentalis Hook. F

Tetrapleura tetraptera Taub.

Uvaria chamae P. Beauv.

Vernonia amygdalina Delile
Poaceae

Arecaceae

Moraceae

Gutiferae

Malvaceae

Convovulaceae

Euphorbiaceae

Anarcadiaceae

Euphorbiaceae

Moringaceae

Musaceae

Lamiaceae

Poaceae

Lauraceae

Euphorbiaceae

Rutaceae

Portulaccaceae

Cucurbitaceae

Leguminosae-Mimosaceae

Annonaceae

Asteraceae
Herb/Grass

Tree

Tree

Tree

Herb

Climber

Shrub

Herb

Tree

Shrub

Tree

Shrub

Shrub

Herb/Grass

Tree

Herb

Tree

Herb

Climber

Tree

Shrub

Shrub

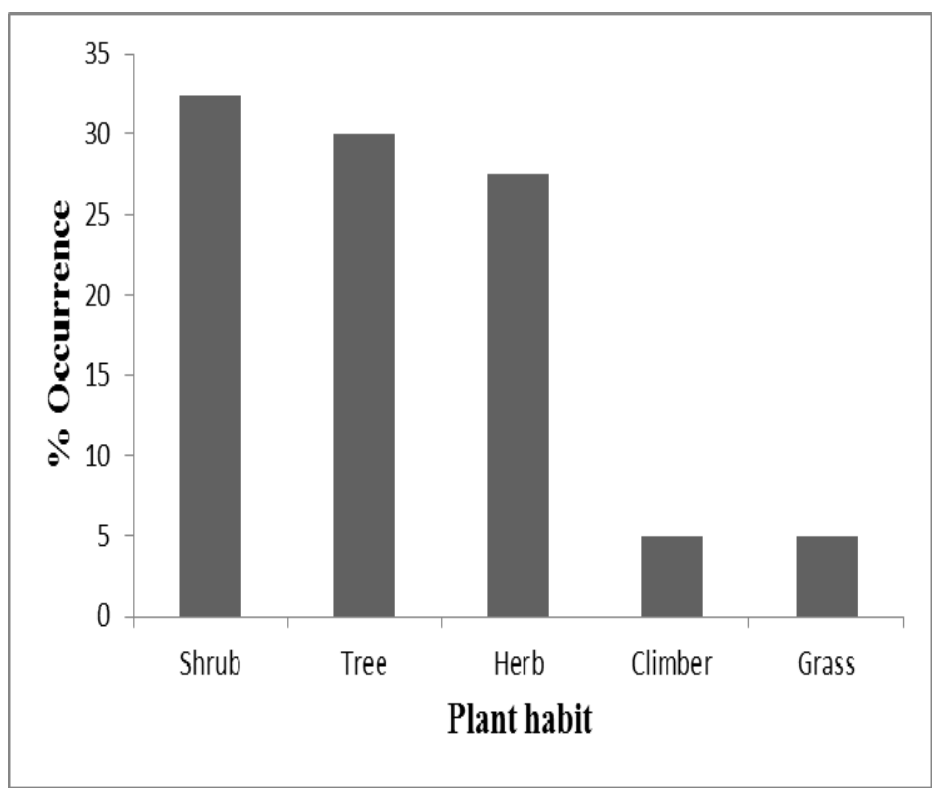

Figure 2. Different plant habits of the medicinal plants mentioned by the students 


\section{Occurrence \%}

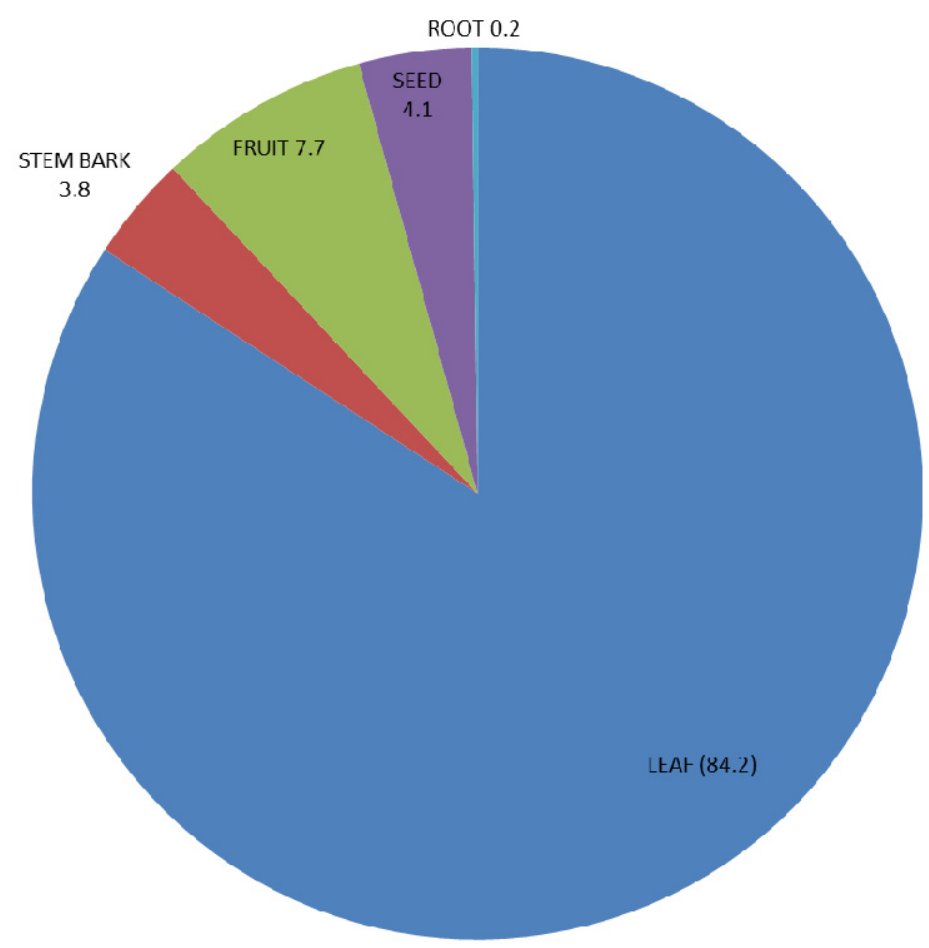

Figure 3. The plant parts used for their medicinal value mentioned by the students

\subsection{Similarity of Use}

Plants like Vernonia amygdalina, Ocimum gratissimum, Carica papaya, Mangifera indica, Citrus x aurantifolia and Azadirachta indica which were mentioned in this study for fever have also been reported in the literature for use as such (Ezekwesili, 2004; Ige, 2011; Challand \& Willcox; 2009). However, the use of Vernonia amygdalina which was the most cited for fever is not very common in most studies in Nigeria (Dike et al., 2012; Obata \& Aigbokhan, 2012). Plants like Mangifera indica and Azadirachta indica are not commonly available in the Niger Delta as in the other parts of Nigeria; this may be the reason for their being less mentioned. Most of the leaves of Azadirachta indica being used for the treatment of malaria are obtained from the markets. Majority of these students have not seen the cultivated tree before.

\subsection{Miscellaneous Sources of Medicines}

A few respondents in addition to various plant species mentioned, cited oil from snakes, raw cray fish, raw eggs and male lizards as sources of medicines. Traditional medicines are diverse health practices, approaches, knowledge and beliefs that incorporate animal in addition to plants (Oreagba \& Oshikoya, 2011).

\subsection{Mode of Preparation of Recipes}

Water is the most used solvent for the preparation of the recipes (Table 9). This confirms the reports of several other authors (Kasali, 2014; Dibong et al., 2011; Saoud et al., 2010). In fact, water is the cheapest and the most available solvent that can dissolve a high number of metabolites and high temperature permits a rapid extraction of active ingredients. However, some of these metabolites can be degraded by heat. Salts were added occasionally probably as a preservative especially when the solvent of preparation was water. Illicit gin was also sometimes used and in this case salt would be unnecessary. 
Table 9. Method of preparation of Plants

\begin{tabular}{|c|c|c|c|c|}
\hline & Plant & Uses & Method of preparation & Mode of application/Dosage \\
\hline \multirow{3}{*}{1} & \multirow{3}{*}{ Bitter leaf } & Fever & Crushed in water/chew leaf & Internal use; 1 shot x 3 till recovery \\
\hline & & Wound & Mix juice with soap & External; x 2 till recovery \\
\hline & & Eye & Crushed and apply juice & External; 1 drop x 2 till recovery \\
\hline 2 & Pawpaw & $\begin{array}{l}\text { Typhoid } \\
\text { fever/malaria }\end{array}$ & Decoction & $\begin{array}{l}\text { Internal; } 1 \text { shotx } 1 \text { for a week/ } \\
\text { Internal; Inhale at night }\end{array}$ \\
\hline 3 & Mango & Malaria fever & Decoction, salt may be added & Internal; 1 shot x 2 for 1 week \\
\hline \multirow{4}{*}{4} & \multirow{4}{*}{ Scent leaf } & Eye & Crushed leaf and apply juice & External; apply as needed \\
\hline & & Wound & $\begin{array}{l}\text { Crushed and apply juice, salt/palm oil } \\
\text { may be added }\end{array}$ & \\
\hline & & Oral use & Concoction & Internal use; 1 shot x 3 till recovery. \\
\hline & & Catarrh & Decoction & Inhalation; at night as needed \\
\hline \multirow{2}{*}{5} & \multirow{2}{*}{ Never die } & Eye & Crushed leaf and apply juice & Eye; 2 drops x 3 \\
\hline & & Stomach ache & Chew & Chew one or two leaves as needed \\
\hline 6 & Lemon grass & Fever & Decoction & 1 shot twice for 1 week \\
\hline 7 & Moringa leaf & Pain & Infusion & $\begin{array}{l}1-2 \text { teaspoonful thrice daily, } \\
1-2 \text { seeds thrice daily }\end{array}$ \\
\hline 8 & $\begin{array}{l}\text { Fluted } \\
\text { pumpkin }\end{array}$ & Blood & $\begin{array}{l}\text { Chew leaf, grind seed in water and } \\
\text { infuse, beverage can be added }\end{array}$ & 1 glass as needed \\
\hline \multirow{3}{*}{9} & \multirow{3}{*}{ Lime } & Malaria & $\begin{array}{l}\text { Extract juice in water/ add pawpaw } \\
\text { leaf, mango stem bark/leaves and boil }\end{array}$ & $\begin{array}{l}\text { External; Bathe as required inhale, I } \\
\text { up twice daily orally }\end{array}$ \\
\hline & & Weight loss & mix juice with honey & $\begin{array}{l}\text { Internal; One glass a day until result } \\
\text { shows }\end{array}$ \\
\hline & & Malaria & Infuse leaf/fruit / lick & $\begin{array}{l}\text { Internal; One glass a day until result } \\
\text { shows }\end{array}$ \\
\hline \multirow[t]{2}{*}{10} & \multirow[t]{2}{*}{$\begin{array}{l}\text { Alligator } \\
\text { pepper }\end{array}$} & Cough & Chew seed & $1-2$ seedsas needed \\
\hline & & Juice/skin & $\begin{array}{l}\text { Add salt to bitter leaf and extract juice } \\
\text { and mix with juice }\end{array}$ & External; Apply twice daily \\
\hline \multirow[t]{3}{*}{11} & \multirow[t]{3}{*}{ Aloe vera } & Oral & Boil leaf & $\begin{array}{l}\text { Internal; } 1 \text { tea cup with corn pap twice } \\
\text { daily }\end{array}$ \\
\hline & & Eye & Squeeze juice & Instil into the eyeonce a day \\
\hline & & Labour induction & Chew & $\begin{array}{l}\text { Internal; Chew leaf once a day for two } \\
\text { weeks }\end{array}$ \\
\hline 12 & Kola nut & Skin infection & Decoction of root & External; Bathe with it twice daily \\
\hline \multirow[t]{2}{*}{13} & Cassava & Pain & Chew leaf with gin & Internal; Chew twice daily \\
\hline & & & Pound fresh leaf with ash & External; Rub twice daily \\
\hline 14 & Plantain & $\begin{array}{l}\text { Chicken } \\
\text { pox/measles }\end{array}$ & Collect sap & $\begin{array}{l}\text { External; Apply twice daily on skin for } \\
\text { measles/ } 1 \text { shot once daily orally }\end{array}$ \\
\hline 15 & Guava & Cough/ulcer & Chew aerial part & Internal; chew 3-4 leaves as needed \\
\hline 16 & Tetraptera & Ulcer & Mix with alligator pepper /Tincture & Internal; 1 shot daily until relief \\
\hline 17 & Orange & Energy/appetite & Add leaf to lime / Decoction & Internal; 1 shot thrice daily \\
\hline 18 & Jute leaf & Skin beauty & Decoction & Internal; Drink like tea \\
\hline
\end{tabular}




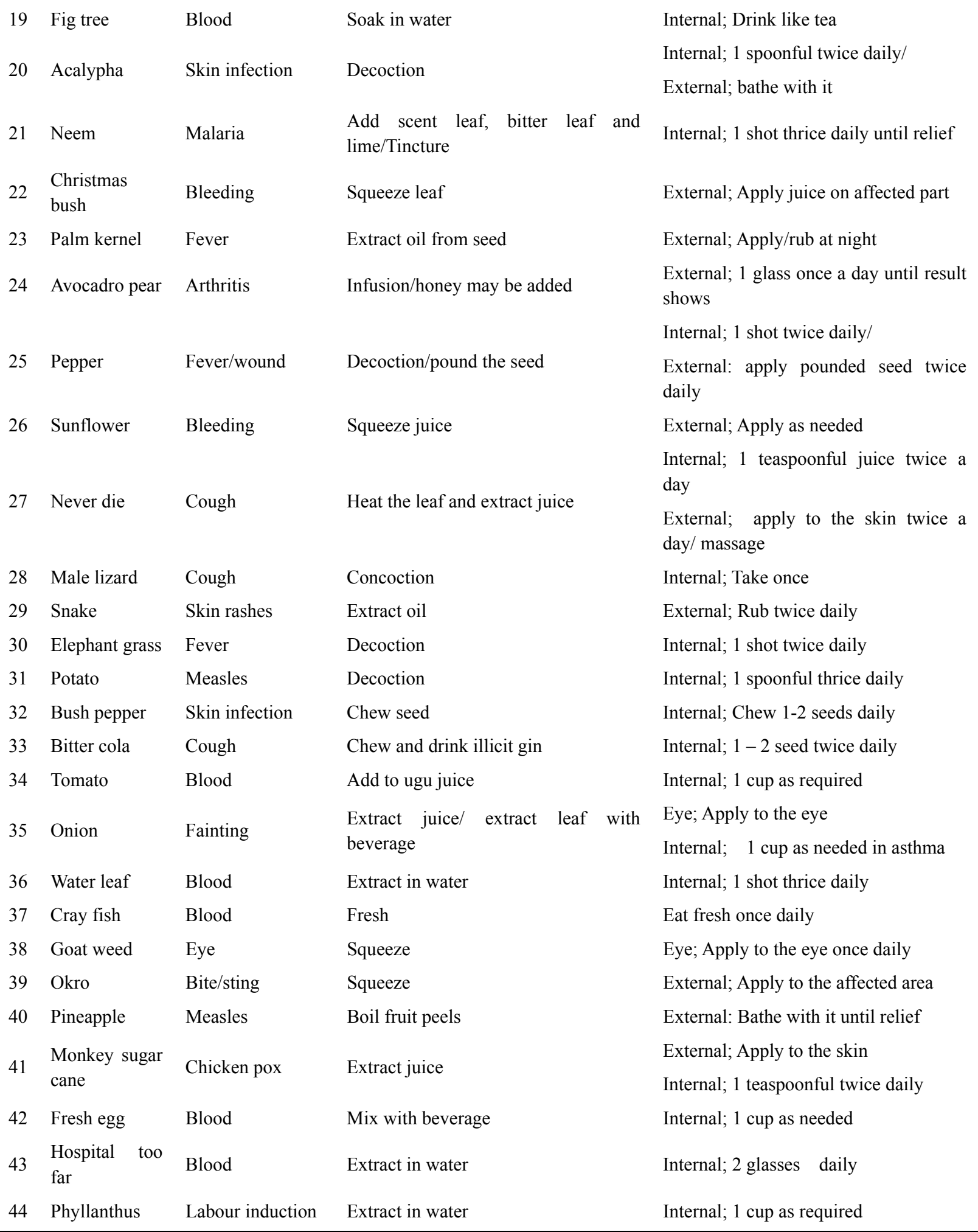

\section{Conclusion}

There is the need to preserve the indigenous knowledge of herbal medicines; this can be done by inclusion of herbal medicine study in school's curriculum. This inclusion will impart the indigenous knowledge in the pupils as they have an advantage of preserving the indigenous knowledge by carrying on the practice of traditional medicine to their old age. Through formal training, herbal medicine will be accorded more recognition and taken more seriously. This will increase awareness in the use of simple, harmless but useful herbs. It will also 
encourage some of the pupils to study herbal medicine or related courses to promote herbal medicine in the country. Now that Nigeria is preparing to legalize herbal medicine to her conventional healthcare system, studying herbal medicine from SS 2 will strengthen the system of medicine which may be incorporated into the healthcare system of the country.

\section{References}

Aguilar, L. (2004). "Biodiversity". Switzerland: IUCN 2004. Retrieved from https://www.cbd.int/gender/doc/fs_uicn_biodiversity.pdf.

Amira, C. O., \& Okubadejo, N. U. (2007). Frequency of complementary and alternative medicine utilization in hypertensive patients attending an urban tertiary care centre in Nigeria. BMC Complementary and Alternative Medicine, 7, 30. http://dx.doi.org/10.1186/1472-6882-7-30

Challand, S., \& Willcox, M. (2009). A clinical trial of the traditional medicine Vernonia amygdalina in the treatment of uncomplicated malaria. Journal of Altern. Complimentary Medicine Nov, 15(11), 1231-1237. http://dx.doi.org/10.1089/acm.2009.0098

Daily Trust Newspaper, Nigeria, Feb. 28, 2012

Danesi, M. A., \& Adetunji, J. B. (1994). Use of alternative medicine by patients with epilepsy: a survey of 265 epileptic patients in a developing country. Epilepsia, 35, 344-351. http://dx.doi.org/10.1111/j.1528-1157.1994.tb02442.x

Deda, P., \& Rubian, R. (2004). Women and biodiversity: The long journey from users to policy-makers. Natural Resources Forum, 28, 201-204.

Dibong, S. D., Mbondo, M. E., Ngoye, A., Kwin, M. F., \& Betti, J. C. (2011). Ethnobotanique etphytomédecine des plantesmédicinales de Douala, Cameroun. Journal of Applied Biosciences, 37, 2496-2507.

Dike, I. P., Obembe, O. O., \& Adebiyi, F. E. (2012). Ethnobotanical survey for potential anti-malarial plants in South-western Nigeria. Journal of Ethnopharmacology. http://dx.doi.org/10.1016/j.jep.2012.10.002

Elujoba, A. A. (1998). Pharmacognostical standardization of herbal medicine: Standardization and utilization of herbal medicine: Challenges of the 21 st century. Proceedings of the 1st International Workshop on Herbal Medicinal Products Nov. 22-24, Ibadan, Nigeria, pp. 30-43.

Ekor, M. (2013). The growing use of herbal medicines: Issues relating to adverse reactions and challenges in monitoring safety. Frontiers in Pharmacology, 4, 177. http://dx.doi.org/103389/phar.2013.00177

Ezekwesili, C. N., Obiorah, K. A., \& Ugwu, O. P. (2004). Evaluation of anti-diarrheal effect of Ocimum gratissimum crude extract on albino rats. Biokemistri., 16(2), 122-131. Retrieved from www.standresjournals.org/journals/SRJMP/pdf/2013/june/Ugonna.pdf

Fakeye, T. O., Adisa, R., \& Musa, I. E. (2009). Attitude and use of herbal medicines among pregnant women in Nigeria. BMC Complementary and Alternative Medicine, 9, 53. http://dx.doi.org/10.1186/1472-6882-9-53

Farnsworth, N. R., \& Morries, R. W. (1976). Higher plants: The sleeping giant of drug development. Am. J. Pharm., 148, 46-52.

Federal Republic of Nigeria. (2007). 2006 Census, Official Gazette No. 24 Vol. 94. Federal Printer, Lagos, Nigeria FGP 71/52007/2, 500 (DL24)

FEPA. (1992). Nigeria: Country study report for Nigeria on costs-benefits and unmet needs of biological diversity conservation. National Biodiversity Unit, Lagos 1992. Retrieved from http://ncseonline.org/PopPlanet/issue.cfm?countryid=2\&iid=2.

Ibrahim, H. A., Imam, I. A., Bello, A. M., Umar, U., Muhammad, S., \& Abdullahi, S. A. (2012). The potential of Nigerian Medicinal Plants as Antimalaria Agent: A Review. International Journal of Science and Technology, 2(8), 600-605.

Idowu, O. A., Soniran, O. T., Ajana, O., \& Aworinde, D. O. (2010). Ethnobotanical survey of antimalarial plants used in Ogun State, Southwest Nigeria. African Journal of Pharmacy and Pharmacology, 4(2), 055-060.

Ige, O. E. (2011). Preliminary Investigation on the Ethnomedicinal Plants of Akoko Division, South West, Nigeria. Global Journal of Health Science, 3(2), 84-89. http://dx.doi.org/10.5539/gjhs.v3n2p84

Kasali, F. M., Mahano1, A. O., Nyakabwa, D. S., Kadima, N. J., Misakabu, F. M., Shibangu, D. S. T., ... Mpiana, P. T. (2014). Ethnopharmacological Survey of Medicinal Plants Used against Malaria in Bukavu City (D. R. Congo). European Journal of Medicinal Plants, 4(1), 29-44. 
Katemo, M., Mpiana, P. T., Mbala, B. M., Mihigo, S. O., Ngbolua, K. N., Tshibangu, D. S. T., \& Koyange, P. R. (2012). Ethnopharmacological survey of plants used against diabetes in Kisangani City (D.R. Congo). $J$. Ethnopharmacol., 144, 39-43. http://dx.doi.org/10.1016/j.jep.2012.08.022

Kunle, O. F., Ali, A. A., \& Egharevba, H. O. (2013). Medicinal Plants Used for the Treatment of Malaria in Rukuba, Bassa Local Government Area of Plateau State, Nigeria. International Journal of Basic and Applied Sciences, 2(4), 134-138.

Lavergne, R., \& Vera, R. (1998). Medicine traditionnelle et pharmacopee etude ethnobotaniquedes plantesutiliseesdan la. pharmacopeetraditinnelle a la Reunion, 41, 24-29. [in French].

Moody, J. O. (2007). Traditional Medicine (pp. 1-6). Paper Delivered at the Mandatory Continuing Professional Development (MCPD) Programme, Module V, Faculty Pharmacy, University of Ibadan, Nov. 21-22.

Moswa, J. L., Ciamala, C., Bongombola, B., Nzigula, N., Kapanda, N., Bokatshinde, O., \& Bunga, M. (2005). Plants used for the treatment of diabetes mellitus in the Democratic Republic ofCongo. Annales de Pharmacie, Presse Universitaire de Kinshasa, 3(1), 87-93.

Nwauzoma, A. B., Magdalene, S., \& Dappa. (2013). Ethnobotanical Studies of Port Harcourt Metropolis, Nigeria. ISRN Botany. Retrieved from http://dx.doi.org/10.1155/2013/829424

Obata, O. O., \& Aigbokhan, E. I. (2012).Ethnobotanical practices among the people of Oka-Akoko, Nigeria. Plant Archives, 12(2), 627-638.

Ogbera, A. O., Dada, O., Adeyeye, F., \& Jewo, P. I. (2010). Complementary and alternative medicine use in diabetes mellitus. West African Journal of Medicine, 29, 158-162.

Omosun, G., Okoro, I. A., Ekundayo, E., Ojimelukwe, P. C., \& Ibe, O. (2013). Ethnobotanical study of medicinal plants useful for malaria therapy in eight local government areas of Abia State, Southeast Nigeria. Advancement in Medicinal Plant Research, 1(2), 39-44.

Oreagba, A. O., Oshikoya, K. A., \& Amachree, M. (2011). Herbal medicine use among urban residents in Lagos, $\begin{array}{lllll}\text { Nigeria. } B M C \text { Complementary and } & \text { Alternative }\end{array}$ http://dx.doi.org/10.1186/1472-6882-11-117

Osemene, K. P., Elujoba, A. A., \& Ilori, M. O. (2011). A Comparative Assessment of Herbal and Orthodox Medicines in Nigeria. Research Journal of Medical Sciences, 5(5), 280-285. http://dx.doi.org/10.3923/rjmsci.2011.280.285

Oshikoya, K. A., Senbanjo, I. O., Njokanma, O. F., \& Soipe, A. (2008). Use of complementary and alternative medicines for children with chronic health conditions in Lagos, Nigeria. BMC Complementary and Alternative Medicine, 8, 66. http://dx.doi.org/10.1186/1472-6882-8-66

Pousset, J. L. (1989). Plantes médicinales africaines. Utilisation pratique.2émè Tome, Paris.French.

Saoud, S., Mohamed, F., Lahce, Z., \& Allal D. (2010). Etudes floristiqueet ethnobotanique des plantes médicinales de la ville de Kenitra (Maroc). Lazaroa, 31, 133-146.

Strgar, J., Pilih, M., Pogačnik, M.,\& Žnidarčič, D. (2013). Knowledge of medicinal plants and their uses among secondary and Grammar school students: A case study from Slovenia. Arch. Biol. Sci., Belgrade, 65(3), 1123-1129.

The Punch Newspaper, Nigeria, October 18th, 2013.

Uzodimma, D. E. (2013). Medico-Ethnobotanical inventory of Ogii, Okigwe Imo State, South Eastern Nigeria I. Global Advanced Research Journal of Medicinal Plants (GARJMP,) 2(2), 030-044.

WHO. (1996). Final report of the seminar on the use of medicinal plants in health care. WHO (WPRO Publication), Tokyo. 
Appendix 1a: Cited plants and the diseases for which they are used

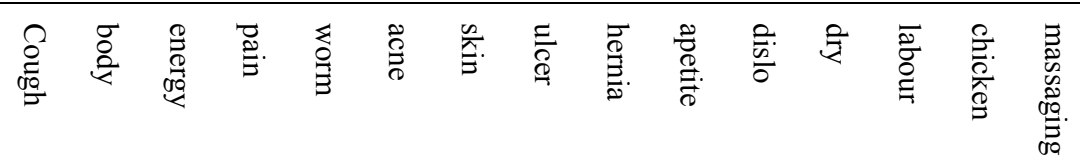

Plant

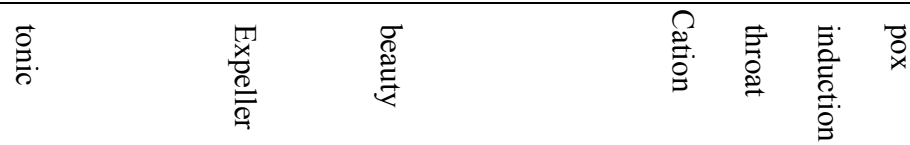

Vernonia amygdalina

Ocimum gratissimum

Carica papaya

11

Mangifera indica

Cymbopogon citratus

Pennisetum purpureum

Citrus $x$ aurantifolia

$\begin{array}{lll}2 & 1 & 2\end{array}$

Psidium guajava

2

Telfairia occidentalis

Aframomum melegueta

Tetrapleura tetraptera

Uvaria chamae

Helianthus anuus

Talinium trangulare

Uvaria chamae

Acalypha wikesiana

Aloe vera

Azadirachta indica

1

1

Chromolaena odorata

Elaeis guineensis

Musa paradisiaca

Citrus $x$ sinensis

Corchorus olitorius

1

2

31

Ficus exasperate

Allium cepa

Manihot esculenta

Hibiscus esculentus

Ananas comosus

Costus afer

2

Garcinia kola

Moringa oleifera

2

Bryophyllum pinnatum

1 2

Ipomea batatas

Ageratum conyzoides

Solanum lycopersicum 


\section{Persea Americana}

Honey

male lizard

4

cray fish

Snake

Cola nitida

fresh egg

Jatropha tanjorensis

phyllanthus amarus

\begin{tabular}{lllllllllllllll}
11 & 2 & 5 & 11 & 1 & 2 & 2 & 6 & 1 & 2 & 1 & 2 & 3 & 3 & 1 \\
\hline
\end{tabular}

Appendix 1b: Cited plants and the diseases for which they are used

\begin{tabular}{lcl}
\hline \multirow{2}{*}{ Plant } & menstrual conjunc asthma fainting bite BP eczema ear navel skin ring \\
& pain $\quad$ tivitis & healing rashes worm \\
\hline
\end{tabular}

Vernonia amygdalina

Ocimum gratissimum

Carica papaya

Mangifera indica

Cymbopogon citratus

Pennisetum purpureum

Citrus $x$ aurantifolia

Psidium guajava

Telfairia occidentalis

Aframomum melegueta

Tetrapleura tetraptera

Uvaria chamae

Helianthus anuus

Talinium trangulare

Uvaria chamae

Acalypha wikesiana

Aloe vera

Azadirachta indica

Chromolaena odorata

Corchorus olitorius

Ficus exasperate

Allium cepa

Manihot esculenta

Ficus exasperate

Allium cepa

12

Manihot esculenta

Hibiscus esculentus

Ananas comosus 
Costus afer

Garcinia kola

Moringa oleifera

2

Bryophyllum pinnatum

$\begin{array}{lll}4 & 1 & 1\end{array}$

Ipomea batatas

Ageratum conyzoides

Solanum lycopersicum

Persea Americana

Honey

male lizard

cray fish

Snake

Cola nitida

fresh egg

Jatropha tanjorensis

phyllanthus amarus

\begin{tabular}{lllllllllllll}
1 & 1 & 1 & 2 & 4 & 3 & & 4 & 2 & 1 & 1 & 1 \\
\hline
\end{tabular}

Appendix 1c: Cited plants and the diseases for which they are used

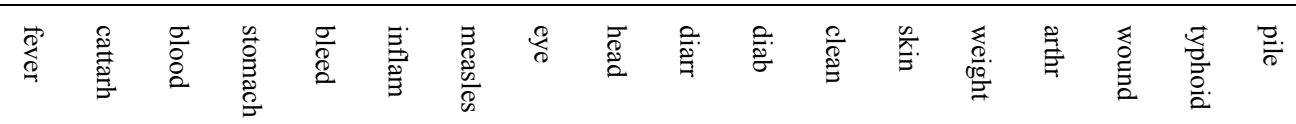

Plant

\begin{tabular}{|c|c|c|c|c|c|c|}
\hline 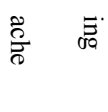 & $\begin{array}{l}\text { : } \\
\stackrel{\stackrel{\Xi}{\Xi}}{\Xi}\end{array}$ & है & $\begin{array}{l}\bar{\delta} \\
\&\end{array}$ & $\frac{8}{8}$ & 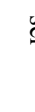 & \\
\hline
\end{tabular}

\begin{tabular}{|c|c|c|c|c|c|c|c|c|c|c|c|c|c|c|c|c|c|c|}
\hline Vernonia amygdalina & 85 & 3 & 1 & 4 & 16 & 1 & 3 & 7 & 2 & 1 & 3 & 2 & & & & & & \\
\hline Ocimum gratissimum & 17 & 2 & & 7 & & & & 25 & 7 & 1 & 1 & & 5 & 1 & 1 & 4 & 1 & 1 \\
\hline Carica papaya & 31 & & & 2 & & & & & & & & & 1 & & & & 6 & \\
\hline Mangifera indica & 15 & & & 1 & & & & & & & & & & & & & & \\
\hline Cymbopogon citratus & 18 & 1 & & 1 & & & & & & & & & & & & & & \\
\hline Pennisetum purpureum & 2 & & & & & & & & & & & & & & & & & \\
\hline Citrus $x$ aurantifolia & 16 & & & 11 & & & 1 & & & & & & & 1 & & & 1 & \\
\hline Psidium guajava & 1 & & & 4 & & & & & & & & & & & & & & \\
\hline Telfairia occidentalis & 1 & & 25 & & & & & & & & & & & & & & & \\
\hline Aframomum melegueta & - & & & & & & & 1 & & & & & 1 & & & & & \\
\hline Tetrapleura tetraptera & - & & & & & & & & & & & & & & & & & \\
\hline Uvaria chamae & 1 & & & & & & & & & & & & & & & 2 & & \\
\hline Helianthus anuus & & & & & 2 & & & & & & & & & & & & & \\
\hline Talinium trangulare & & & 1 & & & & & & & & & & & & & & & \\
\hline Uvaria chamae & & & & & & & & & & & & & 1 & & & & & \\
\hline Acalypha wikesiana & & & & & & & & & & & & & & & & & & \\
\hline
\end{tabular}




\begin{tabular}{|c|c|c|c|c|c|c|c|c|c|c|c|c|c|c|c|c|c|c|}
\hline Aloe vera & 1 & & & & & & & 3 & & & & & 2 & & & & & \\
\hline Azadirachta indica & 12 & & & & & & & & & & & & & & & & 3 & \\
\hline Chromolaena odorata & & & & & 1 & & & & & & & & & & & & & \\
\hline Corchorus olitorius & 1 & & & & & & & & & & & & & & & & & \\
\hline \multicolumn{19}{|l|}{ Ficus exasperate } \\
\hline Allium сера & 1 & & & & & & & & & & & & & & & & & \\
\hline Manihot esculenta & & & & & & & & & & & & & & 1 & & & & \\
\hline Ficus exasperate & & & 1 & & & & & & & & & & & & & & & \\
\hline Allium сера & & & & & & & & 1 & & & & & & & & & & \\
\hline Manihot esculenta & & & & 1 & & 2 & & & & & & & & & & & & \\
\hline \multicolumn{19}{|l|}{ Hibiscus esculentus } \\
\hline Ananas comosus & & & & & & & 1 & & & & & & & & & & & \\
\hline \multicolumn{19}{|l|}{ Costus afer } \\
\hline Garcinia kola & & & & & & 1 & & & & & & & & & & & & \\
\hline Moringa oleifera & & & & 2 & & & & 2 & & & & 2 & & & & & & \\
\hline Bryophyllum pinnatum & & & & & & & & 3 & & & & & & & & & & \\
\hline Ipomea batatas & & & & & & & 1 & & & & & & & & & & & 4 \\
\hline Ageratum conyzoides & & & & & & & & 1 & & & & & & & & & & \\
\hline Solanum lycopersicum & & & 1 & & & & & & & & & & & & & & & \\
\hline Persea Americana & & & & & & & & & & & & & & & 1 & & & \\
\hline Honey & & & & & & & & & & & & & & & 1 & & & \\
\hline \multicolumn{19}{|l|}{ male lizard } \\
\hline cray fish & & & 1 & & & & & & & & & & & & & & & \\
\hline \multicolumn{19}{|l|}{ Snake } \\
\hline Cola nitida & & & & & & & & & & 1 & & & 1 & & & & & \\
\hline fresh egg & & & 1 & & & & & & & & & & & & & & & \\
\hline Jatropha tanjorensis & & & 1 & & & & & & & & & & & & & & & \\
\hline \multicolumn{19}{|l|}{ phyllanthus amarus } \\
\hline & 202 & 6 & 32 & 33 & 19 & 4 & 6 & 43 & 9 & 3 & 4 & 4 & 11 & 3 & 3 & 6 & 11 & 5 \\
\hline
\end{tabular}

\section{Copyrights}

Copyright for this article is retained by the author(s), with first publication rights granted to the journal.

This is an open-access article distributed under the terms and conditions of the Creative Commons Attribution license (http://creativecommons.org/licenses/by/3.0/). 